\title{
Topic of Special Issue: Distributed Diagnosis and Home Healthcare
}

\author{
E. Y. K. Ng • Rajendra Acharya U • Jasjit Suri
}

Published online: 9 June 2010

(C) Springer Science+Business Media, LLC 2010

\section{Foreword}

High-performance computing played a pivotal role in providing convincing solutions to area of healthcare. Signal and Image processing, computer modeling, medical instrumentation, healthcare informatics, communication, biocomputing, and data mining tools have been developed to aid the clinicians in effective diagnosis and treatment.

Distributed Diagnosis and Home Healthcare $\left(\mathrm{D}_{2} \mathrm{H}_{2}\right)$ is new area dealing with miniaturization, integration of instrumentation (wearable and implanted), electronic medicine, inexpensive point-of-care diagnostic systems, biosensors, biomaterials, data acquisition, medical imaging and

\section{E. Y. K. $\mathrm{Ng}(\square)$}

School of Mechanical and Aerospace Engineering,

College of Engineering, Nanyang Technological University,

50, Nanyang Avenue,

Singapore, Singapore 639798

e-mail: mykng@ntu.edu.sg

\section{E. Y. K. Ng}

Office of Biomedical Research,

National University Hospital of Singapore,

Singapore, Singapore

R. Acharya U

Department of Electronics and Computer Engineering,

Ngee Ann Polytechnic,

Singapore, Singapore

J. Suri

Biomedical Technoogies, Inc.,

Denver, CO, USA

e-mail: jsuri@comcast.net

J. Suri

University of Idaho,

Idaho, ID, USA computing. $\mathrm{D}_{2} \mathrm{H}_{2}$ continuously strives to improve the quality of patient care by transforming the delivery of healthcare from a hospital-oriented system to more distributed and home-based. This new approach will help to deliver healthcare facilities to patients anywhere in the world by integrating the information technology with engineering and medical sciences disciplines.

This special issue has two major sections: Section one covers first nine papers and deals with biomedical devices and diagnosis. Section 2 covers the last seven papers and are dedicated to biomedical imaging and diagnosis.

The efficacy of reflected-type green light photoplethysmography (green light PPG) was studied in Paper 1. Transmitted infrared light was used for PPG and the arterial pulse was monitored transcutaneously. The reflected PPG signal contains AC components based on the heartbeatrelated signal from the arterial blood flow and DC components, which include reflectance and scattering from tissue. Generally, changes in AC components are monitored, but the DC components play an important role during heat stress. In this study, authors have compared the signal of green light PPG to infrared PPG and ECG during heat stress. Their results show that, the pulse rates obtained from green light PPG were strongly correlated with the R-R interval of an electrocardiogram in all environments, but those obtained from infrared light PPG displayed a weaker correlation with cold exposure.

Paper 2 deals with an algorithm to extract information from human walking, using Bayesian Network (BN) . Their results show that the $\mathrm{BN}$ extracted from different types of gait measurement data, muscle activities and joint trajectories, could reflect the difference between different walking: the normal walking, simulated hemiplegic walking and paralyzed walking. This approach can be useful for diagnostic, therapeutic, and rehabilitative applications. 
Paper 3 investigates a problem, which is of increasing importance in integrating knowledge in distributed databases for a variety of clinical diagnosis and healthcare applications. Authors propose a new paradigm for integrating frequent item sets discovered from distributed databases and evaluating the results of preservation of unsupervised patterns represented by clusters embedded in these databases. Exhaustive experimentation is performed to evaluate the scalability and robustness of the proposed methodology. The method does not incur the overhead of data integration to perform integrated cluster pattern discovery. Assorted indices of cluster quality assess the accuracy of knowledge merging and the approach clearly preserves cluster quality under a variety of cluster distributions. The method is also robust against the addition of random noise to the data indicating that accurate clusters can be obtained even when simulated acquisition and transmission errors are present. The results are relevant and can be employed in developing semantic relationships mining tool in distributed healthcare data systems.

Paper 4 discusses the possibility of using an Auxiliary Stimulation (AS) to alleviate the symptom of spasticity and muscle fatigue caused by Functional Electrical Stimulation (FES). The results show that AS can enable comparatively stable and durable function restoration assistance. The approach and results shown in this paper are of practical meaning to assistive technology and rehabilitation engineering research area.

Understanding the mechanisms of skin behavior under thermal shock is crucial for medical treatments. However, no reasonable criteria are available for the maximum thermal loadings that skin tissue can survive. Authors have analyzed the behavior (i.e., thermal and neural) of skin tissue exposed to thermal loadings by introducing the thermal shock resistance (a parameter widely used for engineering materials) of skin in Paper 5. Skin thermal shock resistance was analyzed according to two distinct criteria: (i) maximum local temperature at epidermis-dermis (ED) interface defined as the thermal threshold of skin thermal pain; (ii) maximum thermal damage at ED interface defined as the first degree burn where irreversible skin damage occurs. Numerical simulation was performed and the results suggest that the thermal shock resistance of skin tissue depends on the Biot number. These results indicate that skin thermal shock resistance can be used as an efficient tool to predict thermal damage (e.g., burn) and the corresponding pain level induced by noxious thermal loadings.

Level set method was employed to analyze the coupled deformation-flow of individual leukocytes in microfluidic parabolic shear blood flow to understand the physical mechanism of leukocyte separation via microfluidics in Paper 6. Their results show that: (i) Weber number and viscosity ratio have great effects on the deformation of single leukocyte, (ii) difference between the deformation and motion behavior of different subtypes of leukocytes ( $i$. $e$., granulocytes, lymphocytes, monocytes) was observed, and (iii) the existence of a second leukocyte significantly changes the leukocyte deformation and motion. These results shed light on the understanding of the motion and deformation of leukocytes in microchannel flow and provide a theoretical foundation for separating lymphocytes via microfluidics.

Telecare system (TCS) is regarded as one of the important technique in the medical care. ZigBee wireless technique is used to establish a mesh network for monitoring blood pressure automatically and data storage in medical record system for display and further analysis. Moreover, while the blood pressure exceeds the normal range, the system could send a warning signal to remind, or inform the relatives and clinicians in health care center through the personal handy-phone system (PHS) immediately. This proposed system provides an assisted device for monitoring patients' blood pressure during hemodialysis process and is discussed in Paper 7.

Cardiovascular system model provides very useful information about hemodynamic signals. This information may help doctors and researchers to diagnose cardiovascular diseases and develop cardiac assistive devices. A comprehensive cardiovascular system model is developed and an animation of the system is built and explained in Paper 8.

A geometric correction method to restore the image from the mis-alignment distortion was proposed in Paper 9 . Binary tree-based generic demosaicking algorithm to efficiently estimate the missing special components and reconstruct a high-resolution full-spectral image was presented. The effectiveness of geometric correction and demosaicking algorithms to successfully reconstruct a fullspectral image from apparent contrast enhancement between damaged skin and the normal skin was shown.

Atherosclerosis is one of the major risk factors for the onset of cardiovascular diseases. The intima-media thickness (IMT) of the major arteries is a reliable indicator of atherosclerotic progression and a validated marker with prognostic value for cardiovascular pathologies. The manual measurement of IMT carried out by sonographers is time consuming, little repeatable, operator-dependent, and prone to errors. Since early nineties, clinicians benefited from computer systems aiding the IMT measurement. Paper 10 presents a novel technique for carotid arteries highperformance IMT measurement. Three completely automated segmentation strategies are combined following an Inter-Greedy paradigm. The original segmentation techniques are all based on complementary strategies. Their fusion allows for reducing the overall system bias in IMT measurement. The obtained system is still completely user- 
independent and highly accurate: the IMT measurement error is decreased by about $43.5 \% \pm 2.4 \%$ with respect to the initial best performing technique.

An unbiased implicit-reference group-wise (IRG) image registration method is proposed and demonstrated its applications in the construction of a brain white matter fiber tract atlas and the analysis of resting-state functional MRI (fMRI) connectivity in Paper 11. The proposed method jointly estimates transformations, with an elastic deformation model, registering all images to an implicit reference corresponding to the group average. The unbiased registration is applied to build a fiber tract atlas by registering a group of diffusion tensor images. Compared to reference-based registration, the IRG registration improves the fiber track overlap within the group. After applying the method in the fMRI connectivity analysis, results suggest a general improvement in functional connectivity maps at a group level in terms of larger cluster size and higher average t-scores.

Neuropathological studies have revealed an abnormal anatomy of the Corpus Callosum (CC) in autistic brains. A new approach to quantitative analysis of three-dimensional (3D) magnetic resonance images (MRI) of the brain that ensures a more accurate quantification of anatomical differences between the $\mathrm{CC}$ of autistic and normal subjects is proposed in Paper 12. It consists of three main processing steps: (i) segmenting the CC from a given 3D MRI using the learned CC shape and visual appearance; (ii) extracting a centerline of the $\mathrm{CC}$; and (iii) cylindrical mapping of the $\mathrm{CC}$ surface for its comparative analysis. Their experiments revealed significant differences (at the 95\% confidence level) between 17 normal and 17 autistic subjects in four anatomical divisions, i.e. splenium, rostrum, genu and body of their CCs.

Paper 13 deals with the numerical simulation of the behaviour of an anterior cruciate ligament reconstructed knee using interference screw fixation under a single cycle loading or a cyclic loading test and to compare the numerical results with experimental tests using porcine samples of knee joint. A hyper-elastic material model was used to model the tendon graft and evaluate its elongation during continuous and cyclic tensile loading. The rigidity of the interference screw fixation was also examined using the finite element based numerical model.

Functional infrared (IR) imaging is widely adopted in medical field nowadays, with more emphasis on breast cancer and ocular abnormalities. Paper 14 discusses an algorithm to accurately locate the eye and cornea in ocular thermographic sequences, which were recorded utilizing functional infrared imaging. The localization is achieved by snake algorithm coupled with a newly proposed target tracing function. The target tracing function enables automated localization, allows the absence of any manual assistance before the algorithm runs. Genetic algorithm is used to perform the search for global minimum on the function to produce desired localization. The result shows that, in average the region encircled by the algorithm covers $92 \%$ of the true ocular region

Comparison between contra lateral breast images is one of the effective methods in breast cancer detection. Asymmetric temperature distribution can be an indicator of abnormality. The mutual information is a good measure of nonlinear correlation. It is a measure that captures linear and nonlinear dependencies, without requiring the specification of any kind of model of dependence and is discussed in Paper 15 . Therefore, it is suitable to indicate the abnormality. Authors have shown that more similar the thermal image of right and left breast, yielded the normalized mutual information value close to one.

Pulse rates obtained from wearable photoplethysmography (PPG) sensors are important for monitoring cardiovascular condition, especially during exercise. However, it is difficult to precisely count pulse rates during exercise because PPG is sensitive to body movement. The artifacts from body movement are caused by a change in the blood volume at the measurement site, in addition to pulsatile changes. Paper 16 investigates the influence of motion artifact with respect to light source and anatomical sites.

In this special issue, we have made a humble effort to provide various recent progresses in Distributed Diagnosis and Home Healthcare, for the benefit of researchers, professionals and teachers. 